\title{
Elections to the Fellowship, 1995
}

At the meeting of the Court of Electors held on 20 February 1995, the following applicants were approved.

\section{The Fellowship}

Dr N. Ali, Dr H. Al-Kaisi, Lt. Col. R. M. L. Anderson, Dr J. M. Annear, Dr T. E. Baecker, Dr M. G. Barrett, Dr U. B. H. Garuch, Dr D. G. J. Battin, Dr T. S. Bedi, Dr S. M. Benbow, Dr P. J. Beynon, Dr C. E. Boyd, Dr E. F. Bradley, Dr I. Cameron, Dr H. Cheung, Dr J. W. Coid, Dr M. E. Courtney, Dr L. Davidian, Professor T. G. Dinan, Dr R. J. Dolan, Dr G. C. Dunbar, Dr J. M. Eagles, Dr L. F. W. Eickhoff, Dr M. Ellis, Dr N. J. R. Evans, Dr G. S. M. Fitzpatrick, Dr M. D. Gibbs, Dr D. G. Goodhead, Dr G. M. Goodwin, Dr A. K. Gopalaswamy, Dr N. V. Griffin, Dr R. K. Gupta, Dr U. E. Gurisik, Dr H. M. D. Gurling, Dr A. S. Hale, Dr M. J. Harris, Dr J. L. Herzberg, Dr D. Holman, Dr G. M. Holt, Dr M. V. Hynes-O'Sullivan, Dr S. H. Jaigirdar, Dr F. Y. A. Johnson, Dr C. A. Kaplan, Dr T. Kidger, Dr A. C. Knights,
Dr S. V. Korgaonkar, Dr S. Krishnaswamy, Dr D. C. Le Fevre, Dr N. F. N. Loza, Dr S. Luke, Dr O. M. Mahgoub, Dr K. Mak, Dr B. M. Male, Dr R. R. Malik, Dr M. B. Matthews, Professor I. G. McKeith, Dr L. W. Morrish, Dr R. C. O. Mulligan-Rug, Dr J. J. Nehaul, Dr M. Ng, Dr K. R. W. Norton, Dr J. C. O'Grady, Dr A. K. A. Osman, Dr S. E. Proctor, Dr R. W. S. Reid, Dr P. H. Robinson, Dr D. J. Rothery, Dr D. H. Roy, Dr A. J. Rugg, Dr A. W. Scott-Brown, Dr B. S. Sekhawat, Dr P. Sepping, Dr M. F. Shanks, Dr A. M. Sim, Dr I. Singh, Dr P. R. Snowden, Dr G. S. Stein, Dr I. H. Stout, Dr M. M. Sturrock, Dr W. R. Taff, Dr G. V. Tagg, Dr P. F. Thomas, Dr P. Thompson, Dr J. L. Treasure, Wing Cdr. G. J. Turnbull, Dr T. H. Turner, Dr J. Waite, Dr C. A. Walker, Dr A. M. Walsh, Dr P. D. White, Dr F. D. Whitwell, Dr A. J. Williams, Dr S. Wood, Dr Y. O. Younis.

\section{Membership under Bye-Law III 2 (ii)}

Dr A. S. Al Kott, Dr E. Fombonne, Professor R. Green, Dr A. Janca, Dr S. Rajkumar, Professor P. D. Stonier, Professor S. Tyano.

\section{Stage hypnotism}

In response to public concern, the Home Office is conducting a review into the workings of the Hypnotism Act 1952, which regulates performances of stage hypnotism. A small panel nominated by the Royal College of Psychiatrists and the British Psychological Society will assist the review by examining medical evidence of risk of harm to the public from such performances. The Home Office is urgently seeking case notes for consideration by the panel. If you can help, please send anonymised notes to Mrs P. Baskerville, Home Office, Room 722, 50 Queen Anne's Gate, London SW1H 9AT. 